\title{
The eye and thyroid disease
}

\author{
Ajay E Kuriyan, BA ${ }^{1}$, Richard $\mathrm{P}_{\text {Phipps, }} \mathrm{PhD}^{2,3}$, and Steven E Feldon, MD, MBA ${ }^{2}$ \\ ${ }^{1}$ University of Rochester School of Medicine and Dentistry, University of Rochester Medical Center, \\ Rochester, NY 14642, USA \\ ${ }^{2}$ University of Rochester Eye Institute, University of Rochester Medical Center, Rochester, NY \\ 14642, USA \\ ${ }^{3}$ Department of Environmental Medicine, University of Rochester School of Medicine and Dentistry, \\ University of Rochester Medical Center, Rochester, NY 14642, USA
}

\section{Abstract}

Purpose of review-The pathophysiology and optimal management of Thyroid Eye Disease (TED) have not yet been elucidated. Recent studies have increased our knowledge of the disease process and different diagnostic and therapeutic options. This review highlights the recent progress in TED research and identifies areas requiring further advancements.

Recent findings-The pathophysiology of TED likely involves genetic and environmental factors, which may potentiate cellular and humoral-mediated inflammation within the orbit. Despite progress in TED research, a target antigen has not been established with certainty.

New diagnostic methods and questionnaires are being developed that potentially provide information regarding inflammatory activity of TED. Corticosteroids alone or in combination with orbital radiation may be effective in improving TED symptoms. New immunomodulating therapies may also have a role TED management. Surgery is highly effective for treatment of TED-induced optic nerve compression and for managing the chronic soft tissue changes of TED.

Summary-A unifying hypothesis of TED pathophysiology is elusive. Further bench research into the autoimmune process is needed. In addition, large, prospective, randomized clinical trials, based on the inflammatory activity of disease, while difficult to design, are essential to develop a consensus regarding the proper timing and use of anti-inflammatory medications.

\section{Keywords}

Thyroid Eye Disease; Graves' Ophthalmopathy; Graves' Orbitopathy; Thyroid associated ophthalmopathy; Graves' Disease

\section{Introduction}

Thyroid Eye Disease (TED), also called thyroid associated ophthalmopathy (TAO) and Graves' ophthalmopathy/orbitopathy (GO), is the most common orbital disease, affecting 25\%-50\% of patients with Graves' Disease (GD) [1]. Clinical symptoms and signs are usually mild, consisting of ocular irritation with redness and tearing, "stare" due to lid retraction and exophthalmos, and periorbital swelling. Approximately $28 \%$ of TED cases are severe, with

Contact details for corresponding author: Steven E. Feldon, MD, MBA, Phone: (585) 275-1126, Fax: (585) 273-1043, steven_feldon@urmc.rochester.edu, Address: University of Rochester Eye Institute, 210 Crittenden Blvd., Box 659, Rochester, New York 14642.

Conflict of Interest: No conflicting relationships exist. 
restricted motility leading to diplopia, exposure keratopathy, and optic neuropathy [2,3•]. Patients with severe TED may have lower rates of remission of their thyroid disease compared to mild TED patients [4•]. Despite ongoing basic science and clinical research, highly effective therapeutic strategies remain elusive.

\section{Pathophysiology}

In TED, the dramatically enlarged extraocular muscles and increased orbital fat results from a complex interplay among orbital fibroblasts (OF), immune cells, cytokines, autoantibodies, genetics, and environmental factors [1]. However, clear and indisputable identification of a target antigen has not been established.

\section{Autoimmunity}

Lehman and colleagues summarized a plausible pathway for fibroblast activation in TED (Figure 1) [5•]. In this scenario, autoantibodies specific for fibroblast surface thyroidstimulating hormone receptor (TSH-R) and insulin-like growth factor-1 receptor (IGF-1R) are proposed initiators of orbital inflammation [1,5•]. However, increased TSH-R and IGF-1R expression occur with adipogenesis, providing an alternative, non-causative explanation for their presence in TED orbits $[1,6]$.

Autoantibody stimulation activates fibroblasts to release chemokines and cytokines, promoting lymphocyte migration and B cell maturation [5•]. Interaction with humoral and cell-mediated elements of the immune system via OF surface CD40 and human leukocyte antigen-DR (HLADR), may lead to increased autoantigen presentation and further release of cytokines. Prostaglandin $\mathrm{E}_{2}\left(\mathrm{PGE}_{2}\right)$, IL-6, and IL-8 may act on OFs to promote proliferation and production of glycosaminoglycans (GAG), increasing extraorbital muscle mass. Prostaglandin $\mathrm{D}_{2}\left(\mathrm{PGD}_{2}\right)$ and 15 -deoxy- $\Delta 12,14$ - Prostaglandin $\mathrm{J}_{2}\left(15 \mathrm{~d}-\mathrm{PGJ}_{2}\right)$, may act on an OF transcription factor called peroxisome proliferator-activated receptor- $\gamma(\mathrm{PPAR} \gamma)$, leading to OF adipogenesis and increased orbital fat. Cyclooxygenase - 2 (COX-2) expressed by OFs increases synthesis of the involved prostaglandins. Vondrichova and colleagues demonstrated that COX-2 and stearoyl-coenzyme A desaturase (SCD), an adipose tissue marker, are overexpressed in orbital tissue from TED patients with active, compared to chronic, disease $[7 \cdot]$.

In co-cultures, autologous T-lymphocytes stimulate TED OF proliferation [8]. Chen and colleagues demonstrated that there is more infiltration of macrophages and higher mRNA levels of a macrophage chemoattractant called C-C motif chemokine ligand-2 (CCL2)/ monocyte chemoattractant protein-1 (MCP-1) in the orbital fat compared to normal controls $[9 \bullet \bullet]$.

\section{Smoking}

Cigarette smoking is a risk factor for the development and severity of TED [1]. Cawood and colleagues established that OF exposed to cigarette smoke extract (CSE) have a dose-dependent statistically significant increase in GAG production and adipogenesis [10••]. Moreover, IL-1 and CSE had a synergistic effect on adipogenesis.

\section{Genetics}

Bednarczuk and colleagues cited conflicting evidence from case-control studies examining the association between TED and genes involved in inflammation, such as HLA, cytotoxic Tlymphocyte antigen-4 (CTLA-4), tumor necrosis factor (TNF), interferon- $\gamma($ IFN- $\gamma$ ), intercellular adhesion molecule-1 (ICAM-1), and TSH-R [11•]. Based upon these 
investigations, either a unifying polymorphism has yet to be identified or TED has a multifactorial inheritance pattern.

Syed and colleagues found an association between mild/moderate TED and certain single nucleotide polymorphisms (SNPs) in the protein tyrosine phosphatase (PTP) called PTPN12, which interacts with the TSH-R, the proposed target of autoantibodies in GD [12••].

Furthermore, SNPs in the pro-inflammatory IL-23 receptor, which affects T lymphocyte phenotype, were shown to be significantly associated with TED by Huber and colleagues $[13 \bullet \bullet$.

$\mathrm{NF}-\kappa \mathrm{B} 1$, a transcription regulator, is activated by several stimuli, including cytokines. Polymorphisms of NF- $\mathrm{\kappa B} 1$ are associated with several chronic inflammatory and autoimmune diseases [14••]. Kurylowicz and colleagues established a correlation between a polymorphism in the NFKB1 gene promoter ((-94ins/del ATTG) and the development and age of onset of TED within a Japanese cohort [14••].

Chong and colleagues found a positive trend between an IL-13 SNP (2044G/A) and the development of proptosis in a study population of Chinese children [15•]. Boyle and colleagues demonstrated that certain polymorphisms that confer hypersensitivity of the glucocorticoid receptor, which has an anti-inflammatory effect, were associated with less severe TED [16••]. Akaishi and colleagues studied the frequency of the class II major histocompatibility complex (MHC) HLA alleles in 81 Brazilian TED patients and 161 normal controls. TED patients with major extraocular muscle involvement were found to have a higher frequency of the HLADRB1*16 allele, while patients with minor extraocular muscle involvement were found to have a higher frequency of the HLA-DRB $1 * 03$ allele $[17 \bullet \bullet]$.

\section{Clinical Research}

Diagnostic techniques, standardized scoring systems, psychological impact assessment, as well as pharmacological and surgical therapies are areas of clinical TED research.

\section{Diagnostic testing modalities}

Majos and colleagues studied magnetic resonance imaging (MRI) of 40 orbits to examine the correlation between extraocular muscle volume and the measurement of signal intensity (SI) and T2-time (T2-t), which they considered indicators of inflammation [18••]. Both SI and T2$\mathrm{t}$ were found to have statistically significant correlations with volumes of most of the extraocular muscles. This study did not directly correlate the findings of T2-t and SI with clinical symptoms.

Using digital infrared thermal imaging to diagnose early TED, Chang and colleagues studied 32 control orbits and 28 TED orbits. They found a significantly increased temperature among the TED orbits [19••]. A positive correlation was noted between the severity of TED and temperature. Also, a significant decrease in the temperature was found after pulse methylprednisone therapy.

Another tool to measure orbital inflammation is the use of radioactively labeled substances, such as ${ }^{67}$ gadolinium $(\mathrm{Ga})$ citrate and ${ }^{99 \mathrm{~m}}$ technetium (Tc)-labeled agents [20••]. Reyhan and colleagues prospectively studied the use of ${ }^{99 \mathrm{~m}} \mathrm{Tc}(\mathrm{V})$-dimercaptosuccinic acid $(99 \mathrm{mTc}(\mathrm{V})$ DMSA) in 28 TED patients and found a significant correlation between uptake of the agent and Clinical Activity Score (CAS) [20]. Another study by Sun and colleagues demonstrated a correlation between ${ }^{99} \mathrm{~m}$ Tc-HYNIC-Octreotide uptake and CAS in 14 TED patients [21 ••]. This study also showed a significant difference in orbital/occipital uptake ratio between TED patients who were responders to retrobulbar irradiation and those who were not. 


\section{Clinical classification systems}

Severity scores assess TED in a standardized fashion and are especially important in clinical trials. Two such scoring systems are the modified NOSPECS and the CAS systems [22,23]. The modified NOSPECS criteria includes lid retraction, soft tissue inflammation, proptosis, size difference, extraocular muscle involvement, corneal defects, and optic nerve compression [23]. The CAS includes pain, redness, swelling, and impaired visual function, which may signal acute inflammation [22]. Both measure symptom severity.

Dolman and Rootman included subjective and objective components combining aspects of NOSPECS and CAS, with subjective input from the patient about how they feel about their TED status [24]. The so-called VISA classification includes Vision (presence or absence of optic neuropathy), Inflammation (0-8 or mild, moderate, or severe), Strabismus (0-3), restriction (0-3), and Appearance/exposure (mild, moderate, or severe). In future studies, scoring systems should be compared directly to purported objective measures of inflammation, such as the MRI SI and T2-t, thermography, and the radioactive uptake studies described previously.

\section{Psychological and Quality of Life Impact}

Disfigurement and visual changes of TED have a large impact on patients' psychological state and quality of life (QOL). Bradley and colleagues examined the use of the National Eye Institute Visual Function Questionnaire (NEI VQF-25) to assess the effect of TED on QOL in a 30 patient cross-sectional study [25]. Moderate impairments were in the Mental Health and Role Difficulties categories. However, two-thirds of patients felt the questionnaire was not appropriate to assess the impact of TED. Estcourt and colleagues noted development of altered identity in taped, semi-structured interviews of 25 TED patients [26•].

\section{Treatment Trials}

Variation in presentation of TED symptoms and signs, as well as difficulty in assessing autoimmune activity complicates study design. Obstacles include small sample sizes, as well as heterogeneity of response to anti-inflammatory and immunomodulatory therapy.

Corticosteroids-In a review by Rajendram and colleagues, glucocorticosteroid response rate ranges from $63-77 \%$ [27]. Due to their side-effects, use is often limited to three to five months. Disease recurrence after cessation of glucocorticosteroids is common.

A review by Zoumalan and colleagues found that weekly pulse doses of intravenous glucocorticosteroids produced a more favorable response in more patients than oral glucocorticosteroids given daily (74.6 vs. 55.5\%) [28••]. Case reports of severe morbidity or mortality following intravenous glucocorticosteroids treatment for TED have been published, tempering enthusiasm for this route [29,30]. Le Moli and colleagues reported dose-dependent liver damage due to the effect of glucocorticosteroids on hepatocytes [31••].

Radiation-Bradley and colleagues performed a systematic review of nine randomized controlled trials and five observational studies with $>100$ patients on the use of orbital radiation as a treatment for TED [32••]. Based on the randomized controlled trials, orbital radiation did not yield improvement in proptosis, eyelid retraction, nor soft tissue changes. Radiation retinopathy occurred in one to two percent of patients. There was conflicting evidence from the randomized controlled trials regarding the improvement of ocular motility and the one study that examined its effect on QOL had inconclusive findings. However, a meta-analysis by Wei and colleagues, which included data from eight cohort studies and 10 randomized controlled trials, examined the effect of orbital radiation and found that radiation was more effective than a vehicle control and as effective as steroids alone [33•]. The use of combined corticosteroids 
and orbital radiation varied in the studies reviewed by Bradely and colleagues [32••]. Wei and colleagues found radiation and steroid combination therapy to be more effective than either used alone [33•]. Zoumalan and colleagues' observations were similar [28••].

New Immunomodulation Therapies-Etanercept, which has an extracellular binding site for TNF $\alpha$, an inflammatory cytokine, decreases the level of biologically active TNF $\alpha$ [34•]. Possible side-effects include infections, malignancies, and the development of additional autoimmune disorders [34•].

Paridaens and colleagues performed a pilot study using biweekly $25 \mathrm{mg}$ etanercept subcutaneous injections for 12 weeks in 10 euthyroid, recent onset TED patients with a mean pretreatment CAS of 4 (range: 3-6) [34•]. Mean CAS decreased to 2.6 at six weeks and 1.6 at 12 weeks [34•]. No toxicity was noted.

Rituximab is a monoclonal antibody directed against CD20, a B lymphocyte surface antigen [35••]. Possible adverse reactions with rituximab include infusion reaction, serum sickness, and increased infections. Targeting B cells should theoretically eventually dampen the inflammatory process in TED [35••].

Salvi and colleagues reported a steroid refractory case of TED with improvement of CAS after rituximab treatment and no histological evidence of lymphocyte infiltrate in orbital tissue removed during decompression surgery [36]. However, a pilot study of GD patients comparing rituximab and methimazole with methimazole monotherapy by El Fassi and colleagues found no difference in TSH-R antibody levels between the groups [35••].

Rapamycin is an antibiotic in the macrolide class, which also has anti-inflammatory and antifibroblast actions via inhibition of cytokines and growth factor-mediated proliferation of fibroblasts and immune cells [37•]. Possible adverse reactions associated with rapamycin include myelosuppression (most often thrombocytopenia) and hyperlipidemia.

Chang and colleagues reported a case of TED with dysthyroid optic neuropathy that was refractory to steroids and decompression surgery, but improved gradually when treated with rapamycin in conjunction with prednisone taper [37•]. The patient developed hyperlipidemia during rapamycin treatment.

Surgical Therapy-TED surgeries may relieve acute optic nerve compression in dysthyroid optic neuropathy and effectively mitigate the sequelae of chronic orbital inflammation.

Orbital Decompression-Exophthalmos and apical compression of the optic nerve are indications for orbital decompression involving bone. In a study by Jernfors and colleagues, $76 \%$ of patients felt that the surgery was very helpful [38•]. There was minimal improvement in diplopia and late complications included sinusitis (18\%), oroantral fistula (3\%), sensory disturbances (32\%), facial neuralgia (13\%), operation-induced diplopia (8\%), and vision loss (3\%). When comparing the complications (except operation-induced diplopia) to normal, matched controls, only sensory disturbances occurred significantly more frequently.

Fat removal orbital decompression removes orbital contents rather than accommodating them within a larger socket. Richter and colleagues performed a retrospective study of transpalpebral fat removal orbital decompression on 1,374 patients and found a decreased mean proptosis of $5.9 \mathrm{~mm}$, improved symmetry of eye protrusion, improved visual acuity, and improvement in QOL (data unpublished). There were no cases of diplopia postoperatively [39•]. Wu and colleagues performed fat removal orbital decompression on 222 orbits in Taiwan and found a mean postoperative reduction of proptosis by Hertel measurement of $3.6 \mathrm{~mm}$ (range: 1.5 to 
7.5, SD: 1.0) and improved diplopia in $20 \%$ (3/15) of patients with preoperative diplopia $[40 \bullet \bullet$. Only $2.8 \%$ (3/105) of patients developed diplopia postoperatively.

Extraocular Muscle Surgery-Thomas and colleagues, based on previous studies, reported the success rate of extraocular muscle surgery in TED varies from $43 \%$ to $82 \%$ [41•]. Controversy exists as to whether surgical decision-making should be based upon reducing restriction of involved muscles or the degree of deviation between the eyes. In a study of 137 TED patients by Nguyen and colleagues, a higher success rate and lower re-operation rate was found when correcting for the restricted duction compared to deviation [42]. Dal Canto and colleagues confirmed the success of this method in a more recent study of 24 TED patients [43]. On the other hand, Thomas and colleagues found no significant difference in the rate of successful outcomes in a retrospective study of 86 patients comparing the two approaches $(\mathrm{p}=0.55)[41 \bullet]$. Yan and colleagues retrospectively studied 27 TED patients with large angle strabismus $\left(>25^{\circ}\right)$ and found that $74 \%(20 / 27)$ of cases were successfully treated with single extraocular muscle recession, suggesting that for the majority of large angle strabismus, single extraocular muscle surgery is adequate [44•].

\section{Medical and Surgical Recommendations}

On behalf of a European consortium studying TED, Bartalena and colleagues published the set of medical and surgical recommendations shown in Figure $2[45 \bullet, 46]$. They felt that joint management by endocrinology and ophthalmology was optimal.

While many of their suggestions such as cessation of smoking and ocular surface management are common practice, their advocacy of IV steroids, as well as orbital radiation, is controversial and requires further study.

Our approach to management of TED is to use conservative measures for mild to moderate disease directed toward patient comfort. Artificial tears, nocturnal lid taping, elevating the head of the bed and occasional use of diuretics are often sufficient in the active stage. Chronic soft tissue alterations are treated surgically. Severe TED, particularly when accompanied by optic neuropathy, is managed using high dose oral prednisone. If full taper cannot be achieved within 2 months, then bony orbital decompression is performed. Orbital radiation is considered an adjunct to steroids, decompression, or both for recalcitrant disease. Immunosuppressive and immunomodulatory drugs are not generally utilized.

\section{Future Directions}

Understanding the autoimmunity of TED is crucial to improve patient management, especially during active disease. Unfortunately, there are no accepted biomarkers associated with "active" TED, nor are there biomarkers predictive of developing severe disease. Drugs that could suppress early, active TED all have substantial side-effects. Therefore, in the absence of predictive diagnostic or clinical signs, the risk:benefit ratio of treatment may be adverse. Furthermore, many of the inflammatory symptoms and signs may reflect consequences rather than causes of disease. For instance, ocular surface exposure and compartment syndrome may produce substantial non-specific inflammation partially responsive to anti-inflammatory therapy. Anti-fibrotic agents may prove more effective than immune suppressant therapy late in the disease process.

Questionnaires designed to assess autoimmune inflammatory activity must be validated against objective measures of inflammation. In the future blood tests looking at inflammatory markers of TED may prove highly beneficial in planning therapy. Soluble CD40, CD154, vascular cell adhesion molecule- 1 , and matrix metalloproteinase- 9 or urine tests for oxidative stress, such as urinary 8 -OHdG levels, are possible examples [47•,48•,49•]. 


\section{Conclusion}

Great strides have been made over the past year in many aspects of TED research, especially regarding the pathophysiology of TED, assessment of inflammatory activity, and novel uses of immunomodulating therapies. New medical and surgical management options have been introduced, while traditional therapies have been further studied. Much research remains to be done in the field of TED pathophysiology in order to provide insight regarding risk factors, targets for therapy, and the ideal window for anti-inflammatory versus anti-fibrotic treatments. Large, prospective, randomized, controlled clinical trials must be designed around proper assessment of subjects' inflammatory status. These studies will lead to a consensus regarding management of TED.

\section{Acknowledgments}

Financial Support: ES01247, EY017123, EY011708, and Research to Prevent Blindness (Challenge Grant)

\section{References and recommended readings}

Papers of particular interest, published within the annual period of review, have been highlighted as:

- Of special interest

- Of outstanding interest

1. Garrity JA, Bahn RS. Pathogenesis of Graves Ophthalmopathy: Implications for Prediction, Prevention, and Treatment. American Journal of Ophthalmology 2006;142:147-147. [PubMed: 16815265]

2. Prummel M, Bakker A, Wiersinga W, et al. Multi-center study on the characteristics and treatment strategies of patients with Graves' orbitopathy: the first European Group on Graves' Orbitopathy experience. Eur J Endocrinol 2003;148:491-495. [PubMed: 12720530]

-3. Schotthoefer EO, Wallace DK. Strabismus associated with thyroid eye disease. Curr Opin Ophthalmol 2007;18:361-365. [PubMed: 17700227]This article reviews methods for the treatment and evaluation of strabismus associated with TED, with a focus on the developments in 2006.

-4. Eckstein AK, Lax H, Losch C, et al. Patients with severe Graves' ophthalmopathy have a higher risk of relapsing hyperthyroidism and are unlikely to remain in remission. Clin Endocrinol 2007;67:607612.This retrospective observational study of 158 TED patients reports that patients with more severe TED and with higher levels of TSH-R autoantibodies were less likely to go into remission.

•5. Lehmann GM, Garcia-Bates TM, Smith TJ, et al. Regulation of Lymphocyte Function by PPARgamma: Relevance to Thyroid Eye Disease-Related Inflammation. PPAR Res 2008;2008:895.This article reviews TED pathophysiology, with an emphasis on PPAR $\gamma$ 's potential dual anti-inflammatory and pro-adipogenic roles.

6. Entingh-Pearsall A, Kahn CR. Differential roles of the insulin and insulin-like growth factor-I (IGFI) receptors in response to insulin and IGF-I. J Biol Chem 2004;279:38016-38024. [PubMed: 15247278]

-7. Vondrichova T. COX-2 and SCD, markers of inflammation and adipogenesis, are related to disease activity in Graves' ophthalmopathy. Thyroid 2007;17:511. [PubMed: 17614770]A study of the difference in levels of COX-2 and SCD gene expression in orbital adipose/connective tissue between acute and chronic phase TED patients demonstrated increased levels of COX-2 and SCD gene expression among acute phase patients. Diclofenac, a COX-2 inhibitor and PPAR $\gamma$ antagonist, reduced adipogenesis in vitro and requires further study.

8. Feldon SE, Park DJ, O'Loughlin CW, et al. Autologous T-lymphocytes stimulate proliferation of orbital fibroblasts derived from patients with Graves' ophthalmopathy. Invest Ophthalmol Vis Sci 2005;46:3913-3921. [PubMed: 16249464] 
••9. Chen M, Chen M, Liao S, et al. Role of macrophage infiltration in the orbital fat of patients with Graves' ophthalmopathy. Clin Endocrinol (OnlineAccepted articles). 10.1111/j. 1365-2265.2008.03219.xUsing orbital adipose tissue of 15 TED and six controls patients, this study demonstrated increased expression of MCP-1 and macrophage infiltration within adipose tissue, but not connective tissue. The authors conclude that macrophages may play an important role in the adipogenic process of TED.

$\bullet$ 10. Cawood TJ, Moriarty P, O'Farrelly C, et al. Smoking and thyroid-associated ophthalmopathy: A novel explanation of the biological link. J Clin Endocrinol Metab 2007;92:59-64. [PubMed: 17047020]A study of the effects of cigarette smoke extract +/- IL-1 on ICAM-1, GAG production, and adipogenesis in orbital tissue from 10 TED patients and nine controls. The authors found a dose-dependent relationship between CSE and both GAG production and adipogenesis, but no effect on ICAM-1 expression. Furthermore, IL-1 and CSE had a synergistic effect on adipogenesis, which was reduced by $81 \%$ with an anti-IL- 1 antibody. The authors suggest IL- 1 as a possible therapeutic target.

-11. Bednarczuk T, Gopinath B, Ploski R, et al. Susceptibility genes in Graves' ophthalmopathy: searching for a needle in a haystack? Clin Endocrinol (Oxf) 2007;67:3-19. [PubMed: 17521325]This review article examines several potential loci in candidate genes, which may increase susceptibility to TED. The studies were considered to be inconclusive. Suggestions were made for the design of future genetic studies.

$\bullet$ 12. Syed AA, Simmonds MJ, Brand OJ, et al. Preliminary evidence for interaction of PTPN12 polymorphism with TSHR genotype and association with Graves' ophthalmopathy. Clin Endocrinol (Oxf) 2007;67:663-667. [PubMed: 17608818]A study of the relationship between seven SNPs of the PTPN12 gene and the development of GD in 1,058 GD patients and 864 controls demonstrated no association between the SNPs and the development of GD. However, three SNPs were associated with the development of TED and four SNPs revealed evidence of interaction with the TSH-R. Larger studies are required to confirm these preliminary findings.

$\bullet$ 13. Huber AK, Jacobson EM, Jazdzewski K, et al. Interleukin (IL)-23 receptor is a major susceptibility gene for Graves' ophthalmopathy: the IL-23/T-helper 17 axis extends to thyroid autoimmunity. $\mathbf{J}$ Clin Endocrinol Metab 2008;93:1077-1081. [PubMed: 18073300]The association between four SNPs of the IL-23R gene and development of TED and GD was studied in 216 TED patients and 368 controls. Two SNPs were associated with development of TED and GD and one SNP was associated with GD development alone. These were considered as potentially proinflammatory SNPs, contributing to the development of TED.

••14. Kurylowicz A, Hiromatsu Y, Jurecka-Lubieniecka B, et al. Association of NFKB1 -94ins/del ATTG promoter polymorphism with susceptibility to and phenotype of Graves' disease. Genes Immun 2007;8:532-538. [PubMed: 17690684]The association between the NFkB1 -94ins/del ATTG promoter polymorphism and GD was studied in 586 GD and 882 control Polish patients and 424 GD and 222 control Japanese patients. There was a correlation between the polymorphism and both the development and age of onset of TED within the Japanese cohort. This polymorphism was associated with other chronic inflammatory diseases, possibly contributing to TED's comorbidity with other autoimmune disorders.

-15. Chong KK, Chiang SW, Wong GW, et al. Association of CTLA-4, IL-13 Gene polymorphism with Graves' disease and ophthalmopathy in Chinese children. Invest Ophthalmol Vis Sci. 2008A study examining the relationship between polymorphisms of CTLA-4 and IL-13 genes and the development of GD and TED in 177 GD and 151 control Chinese children did not find a significant association, but did find a trend between the IL-13 SNP, 2044G/A (R130Q), and proptosis.

• 16. Boyle B, Koranyi K, Patocs A, et al. Polymorphisms of the glucocorticoid receptor gene in Graves ophthalmopathy. Br J Ophthalmol 2008;92:131-134. [PubMed: 18156379]No association between polymorphisms of the glucocorticoid receptor gene and the development and clinical phenotype of TED was established in a study of 95 TED and 160 control Hungarian patients. However, the BclI polymorphism was significantly more frequent in patients with milder TED, which the authors attributed to increased glucocorticoid receptor signaling. This polymorphism may contribute to variable responses to corticosteroid therapy.

$\bullet$ 17. Akaishi PM, Cruz AA, Silva FL, et al. The role of major histocompatibility complex alleles in the susceptibility of Brazilian patients to develop the myogenic type of Graves' orbitopathy. Thyroid 2008;18:443-447. [PubMed: 18352820]A study of 81 TED and 161 control Brazilian patients found 
that patients with muscle involvement had a higher frequency of HLA-DRB1*16, while patients with minimal muscle involvement had a higher frequency of HLA-DRB $1 * 03$. There may be a genetic predisposition for extraocular muscle involvement in TED.

••18. Majos A, Pajak M, Grzelak P, et al. Magnetic Resonance evaluation of disease activity in Graves' ophthalmopathy: T2-time and signal intensity of extraocular muscles. Med Sci Monit 2007;13:4448. [PubMed: 17507884]A study of MRIs of 40 orbits demonstrated a correlation between both signal intensity and T2-time and the muscle volume of the superior, inferior, and medial recti. The T2-time generally had a stronger correlation. Further testing directly correlating MRI findings with patient symptoms are needed to establish signal intensity and T2-time as measures of disease activity.

$\bullet$ 19. Chang TC, Hsiao YL, Liao SL. Application of digital infrared thermal imaging in determining inflammatory state and follow-up effect of methylprednisolone pulse therapy in patients with Graves' ophthalmopathy. Graefes Arch Clin Exp Ophthalmol 2008;246:45-49. [PubMed: $17653750]$ A study of the ability of thermography to assess TED inflammatory activity and response to treatment found a positive correlation between disease severity and temperature and a significant decrease in temperatures after pulse methylprednisone therapy.

••20. Reyhan M, Toygar O, Sukan A, et al. 99mTc(V)-DMSA SPECT for the assessment of disease activity in Graves' ophthalmopathy. Nucl Med Commun 2007;28:775-781. [PubMed: 17728607] The use of ${ }^{99 \mathrm{~m}} \mathrm{Tc}(\mathrm{V})$-dimercaptosuccinic acid scintigraphy to assess TED activity was studied in eight clinically active patients and 20 clinically inactive patients. The authors found that uptake of the agent was significantly correlated with clinical activity.

••21. Sun H, Jiang XF, Wang S, et al. (99m)Tc-HYNIC-TOC scintigraphy in evaluation of active Graves' ophthalmopathy (GO). Endocrine 2007;31:305-310. [PubMed: 17906380]The use of ${ }^{99 \mathrm{~m}} \mathrm{Tc}-$ HYNIC-Octreotide orbital/occipital uptake ratio in 14 TED patients demonstrated a significant correlation between CAS and the orbital/occipital uptake ratio. Furthermore, a higher uptake ratio was found among responders to irradiation. Further studies of ${ }^{99} \mathrm{~m}$ Tc-HYNIC-Octreotide are needed to establish it as a tool in assessing TED severity and response to treatment.

22. Mourits MP, Koornneef L, Wiersinga WM, et al. Clinical criteria for the assessment of disease activity in Graves' ophthalmopathy: a novel approach. Br J Ophthalmol 1989;73:639-644. [PubMed: 2765444]

23. Wiersinga WM, Prummel MF, Mourits MP, et al. Classification of the eye changes of Graves' disease. Thyroid 1991;1:357-360. [PubMed: 1841734]

24. Dolman PJ, Rootman J. VISA Classification for Graves orbitopathy. Ophthal Plast Reconstr Surg 2006;22:319-324.

25. Bradley EA, Sloan JA, Novotny PJ, et al. Evaluation of the National Eye Institute visual function questionnaire in Graves' ophthalmopathy. Ophthalmology 2006;113:1450-1454. [PubMed: 16769116]

-26. Estcourt S, Vaidya B, Quinn A, et al. The impact of thyroid eye disease upon patients' wellbeing: a qualitative analysis. Clin Endocrinol (Oxf) 2008;68:635-639. [PubMed: 17973942]Taped, semistructured interviews of 25 TED patients revealed that the physical manifestations of TED led to patients developing an altered sense of identity, which impacted other domains of life.

27. Rajendram R, Lee RW, Potts MJ, et al. Protocol for the combined immunosuppression \& radiotherapy in thyroid eye disease (CIRTED) trial: A multi-centre, double-masked, factorial randomised controlled trial. Trials 2008;9:6. [PubMed: 18237441]

$\bullet$ 28. Zoumalan CI, Cockerham KP, Turbin RE, et al. Efficacy of corticosteroids and external beam radiation in the management of moderate to severe thyroid eye disease. J Neuroophthalmol 2007;27:205-214. [PubMed: 17895822]This article reviewed studies on the use of corticosteroids and orbital radiation for TED management. The authors found combined corticosteroid and orbital radiation therapy was more effective than oral corticosteroid monotherapy. Weekly pulse IV doses of corticosteroids were found to be more effective and had fewer side-effects than oral corticosteroids.

29. Gursoy A, Cesur M, Erdogan MF, et al. New-onset acute heart failure after intravenous glucocorticoid pulse therapy in a patient with Graves' ophthalmopathy. Endocrine 2006;29:513-516. [PubMed: 16943591] 
30. Weissel M, Hauff W. Fatal liver failure after high-dose glucocorticoid pulse therapy in a patient with severe thyroid eye disease. Thyroid 2000;10:521. [PubMed: 10907999]

••31. Le Moli R, Baldeschi L, Saeed P, et al. Determinants of liver damage associated with intravenous methylprednisolone pulse therapy in Graves' ophthalmopathy. Thyroid 2007;17:357-362. [PubMed: 17465867]A prospective observational study comparing the hepatotoxic effects of highdose IV methylprednisone and low-dose IV methylprednisone in 27 TED patients found dosedependent liver damage caused by the toxic effects of glucocorticoids on hepatocytes. The authors concluded that IV methylprednisone could be safely administered if the cumulative dose is $<8$ grams and liver function is monitored.

••32. Bradley EA, Gower EW, Bradley DJ, et al. Orbital radiation for graves ophthalmopathy: a report by the American Academy of Ophthalmology. Ophthalmology 2008;115:398-409. [PubMed: 18082885]Five observational studies and nine randomized controlled trials were reviewed to assess orbital radiation treatment for TED. While three of five observational studies found favorable outcomes for $40 \%-97 \%$ of patients, the was no Level I evidence demonstrating that orbital radiation led to an improvement in proptosis, eyelid retraction, or soft tissue changes. The heterogeneity of the studies reviewed limited the authors' ability to assess the effect of orbital radiation.

-33. Wei RL, Cheng JW, Cai JP. The use of orbital radiotherapy for Graves' ophthalmopathy: quantitative review of the evidence. Ophthalmologica 2008;222:27-31. [PubMed: 18097177]A meta-analysis that established orbital radiation and corticosteroid combination therapy was more effective than orbital radiation alone. Orbital radiation was found to be as effective as oral corticosteroids and significantly more effective than a vehicle control. Corticosteroids were more effective when administered intravenously than orally.

-34. Paridaens D, van den Bosch WA, van der Loos TL, et al. The effect of etanercept on Graves' ophthalmopathy: a pilot study. Eye 2005;19:1286-1289. [PubMed: 15550932]The use of etanercept in 10 consecutive TED patients resulted in improvement in disease severity after 12 weeks of treatment, without any major side effects during the follow-up period.

••35. El Fassi D, Nielsen CH, Bonnema SJ, et al. B lymphocyte depletion with the monoclonal antibody rituximab in Graves' disease: a controlled pilot study. J Clin Endocrinol Metab 2007;92:1769-1772. [PubMed: 17284622]A prospective, controlled, non-randomized study demonstrated higher rate of remission of GD among patients treated with rituximab. However, no significant difference in levels of autoantibodies was observed in rituximab treated patients. The authors concluded that the high cost, low efficacy, and potential side-effects were barriers to the use of rituximab in uncomplicated GD.

36. Salvi M, Vannucchi G, Campi I, et al. Efficacy of rituximab treatment for thyroid-associated ophthalmopathy as a result of intraorbital B-cell depletion in one patient unresponsive to steroid immunosuppression. Eur J Endocrinol 2006;154:511-517. [PubMed: 16556712]

-37. Chang S, Perry JD, Kosmorsky GS, et al. Rapamycin for treatment of refractory dysthyroid compressive optic neuropathy. Ophthal Plast Reconstr Surg 2007;23:225-226.The authors report the use of rapamycin in a patient with dysthyroid optic neuropathy refractory to steroids and orbital decompression Improvements in visual acuity, color vision, and visual fields were noted.

-38. Jernfors M, Valimaki MJ, Setala K, et al. Efficacy and safety of orbital decompression in treatment of thyroid-associated ophthalmopathy: long-term follow-up of 78 patients. Clin Endocrinol (Oxf) 2007;67:101-107. [PubMed: 17466006] Orbital decompression surgery in 78 TED patients demonstrated decompression surgery could produce long-term improvement in measurements of proptosis and visual acuity in many patients, but did not improve measurements to the level of matched controls.

-39. Wu CH, Chang TC, Liao SL. Results and predictability of fat-removal orbital decompression for disfiguring graves exophthalmos in an asian patient population. Am J Ophthalmol 2008;145:755759. [PubMed: 18241831]A cross-sectional study of fat-removal orbital decompression surgery in 222 TED orbits demonstrated reduction of proptosis and improvement of diplopia in $20 \%$ of patients, with a $2.8 \%$ rate of post-surgical diplopia. Based on volume of fat removed during surgeries and the resulting Hertel changes, using multivariate analysis, a formula was developed to predict the change in proptosis, which may be helpful for surgical decision-making.

••40. Richter DF, Stoff A, Olivari N. Transpalpebral decompression of endocrine ophthalmopathy by intraorbital fat removal (Olivari technique): experience and progression after more than 3000 operations over 20 years. Plast Reconstr Surg 2007;120:109-123. [PubMed: 17572552]In a 
retrospective study of transpalpebral fat removal decompression surgery on 1,374 TED patients, TED symptoms improved and no cases of postoperative diplopia were reported. The authors recommended this technique as a viable surgical option for TED soft-tissue complications.

-41. Thomas SM, Cruz OA. Comparison of two different surgical techniques for the treatment of strabismus in dysthyroid ophthalmopathy. J AAPOS 2007;11:258-261. [PubMed: 17572340]A retrospective study of extraocular muscle surgery for strabismus associated TED demonstrated no difference in outcomes with correction of the restricted duction or correction for degree of deviation in 52 patients. These findings contradicted the findings of previous studies that demonstrated improved outcomes with correction for the restricted duction.

42. Nguyen VT, Park DJ, Levin L, et al. Correction of restricted extraocular muscle motility in surgical management of strabismus in graves' ophthalmopathy. Ophthalmology 2002;109:384-388. [PubMed: 11825827]

43. Dal Canto AJ, Crowe S, Perry JD, et al. Intraoperative relaxed muscle positioning technique for strabismus repair in thyroid eye disease. Ophthalmology 2006;113:2324-2330. [PubMed: 17157137]

-44. Yan J, Zhang H. The surgical management of strabismus with large angle in patients with Graves' ophthalmopathy. Int Ophthalmol 2008;28:75-82. [PubMed: 17611721]A retrospective study of surgical management of large deviation ( $>/=25$ degrees) in 27 patients with strabismus associated with TED found favorable outcomes and low re-operation rates. The authors found that surgery involving one rectus muscle was adequate in the majority of cases.

• 45. Bartalena L, Baldeschi L, Dickinson A, et al. Consensus statement of the European Group on Graves' orbitopathy (EUGOGO) on management of GO. Eur J Endocrinol 2008;158:273-285. [PubMed: 18299459]This is a consensus statement, based on current research, on the management of TED by the European Group on Graves' Orbitopathy. The recommendations are graded following the guidelines of the Agency for Healthcare Research and Quality. They are defined for different levels of severity and complications of TED.

46. Wiersinga WM. Management of Graves' ophthalmopathy. Nat Clin Pract Endocrinol Metab 2007;3:396-404. [PubMed: 17452966]

-47. Mysliwiec J, Adamczyk M, Pawlowski P, et al. Serum gelatinases (MMP-2 and MMP-9) and VCAM-1 as a guideline in a therapeutic approach in Graves' ophthalmopathy. Endokrynologia Polska 2007;58:105-109. [PubMed: 17578825]This study demonstrated higher MMP-9 levels in TED patients and higher VCAM-1 levels among corticosteroid-responders than non-responders.

-48. Tsai CC, Kao SC, Cheng CY, et al. Oxidative stress change by systemic corticosteroid treatment among patients having active graves ophthalmopathy. Arch Ophthalmol 2007;125:1652-1656. [PubMed: 18071117]Levels of the oxidative stress marker, 8-hydroxy-2'-deoxyguanosine (8$\mathrm{OHdG})$, were elevated in patients with active TED and decreased after corticosteroid treatment.

-49. Mysliwiec J, Waligorski D, Nikolajuk A, et al. Soluble CD40 and its ligand CD154 in patients with Graves' ophthalmopathy during combined therapy with corticosteroids and teleradiotherapy. Adv Med Sci 2007;52:104-108. [PubMed: 18217399]A study of soluble CD40 and CD154 measurements as markers of TED in 51 TED patients and 10 controls demonstrated that serum CD40 and CD154 were elevated in TED patients. Non-responders to IV methylprednisone were found to have significantly elevated CD40/CD154 ratios after treatment and at the end of the study. 
Autoantibody

(Anti-TSH receptor, Anti-IGF-1 receptor)

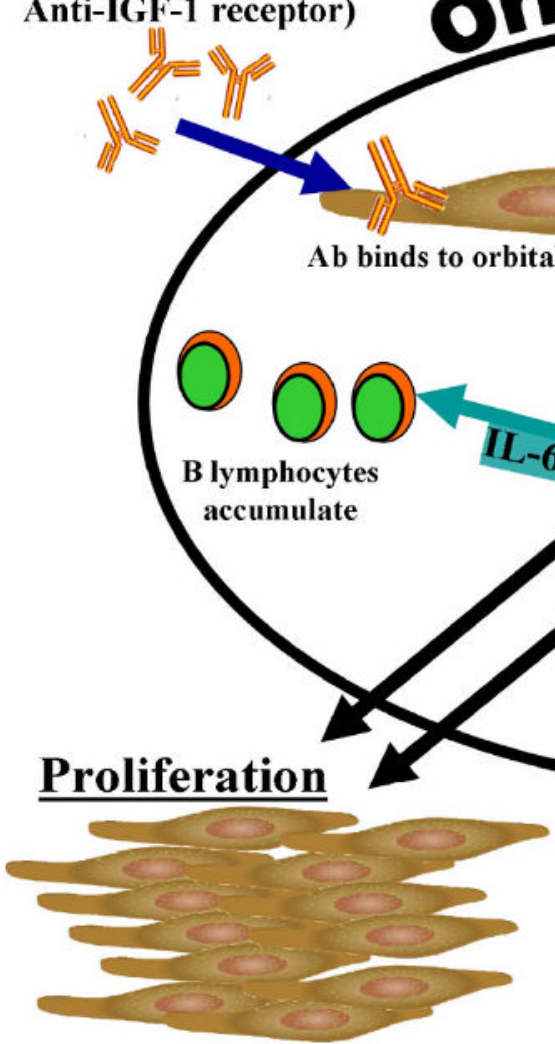

activation increases autoantigen presentation $N=$

Figure 1. Pathophysiology of TED

According to one current model, TED is triggered by binding and activation of orbital fibroblasts by autoantibodies. These autoantibodies could be specific for antigens such as TSH$\mathrm{R}$ and/or IGF-1R. Activated orbital fibroblasts release chemokines, including IL-16, RANTES, and CXCL10, which recruit $\mathrm{T}$ lymphocytes into the orbit. These lymphocytes then interact with fibroblasts, potentially activating each other, further promoting cytokine production (IFN $\gamma, \mathrm{TNF}_{\alpha}, \mathrm{PGD}_{2}$, and $15 \mathrm{~d}-\mathrm{PGJ}_{2}$ ) and secretion of $\mathrm{T}$ cell-activating factors by the fibroblasts (IL-8 and CXCL10). Fibroblasts are also stimulated to secrete IL-6 (promoting B cell differentiation) and to increase autoantigen presentation, both of which amplify the overall response. The interactions of fibroblasts with $\mathrm{T}$ cells result in the deposition of extracellular matrix molecules, fibroblast proliferation, and fat accumulation.

See Lehmann, GM [5]. 


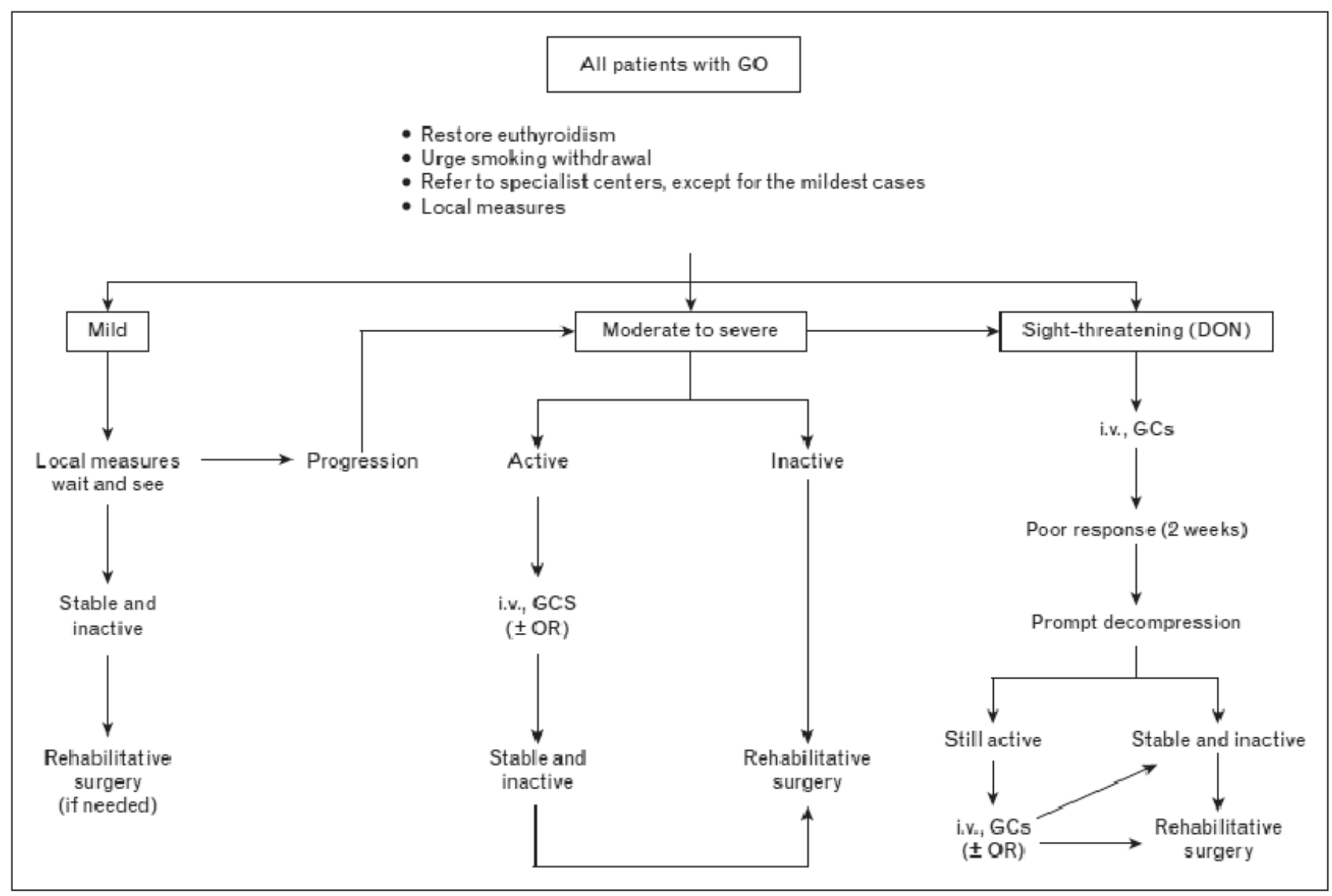

Figure 2. Management of Graves' orbitopathy

Rehabilitative surgery includes orbital decompression, squint surgery, lid lengthening, and blepharoplasty/browplasty. i.v. GCs, intravenous glucocorticoids; OR, orbital radiotherapy; DON, dysthyroid optic neuropathy.

See Bartalena L. [45] 